\title{
Differences in Sensitivity to New Therapies Between Primary and Metastatic Breast Cancer: A Need to Stratify the Tumor Response?
}

Hubert Beaumont ( $\square$ Hubert.beaumont@mediantechnologies.com )

MEDIAN Technologies https://orcid.org/0000-0002-7624-8956

Nathalie Faye

Median Technologies

Antoine lannessi

Antoine-Lacassagne Centre: Centre Antoine-Lacassagne

Emmanuel Chamorey

Centre Antoine-Lacassagne

\section{Catherine Kliffa}

Median Technologies

Chih-Yi Hsieh

Aslan Pharmaceuticals

Research article

Keywords: Breast Neoplasms, Tumor burden, Multidetector Computed Tomography, biomarkers

Posted Date: October 19th, 2020

DOl: https://doi.org/10.21203/rs.3.rs-92580/v1

License: (9) (i) This work is licensed under a Creative Commons Attribution 4.0 International License.

Read Full License 


\section{Abstract}

Objective: We compared therapeutic response of Varlitinib + Capecitabine (VC) versus Lapatinib + Capecitabine (LC) in patients with human epidermal growth factor receptor 2-positive metastatic breast cancer after trastuzumab therapy by assessing changes in target lesion $(\mathrm{TL})$ diameter and volume per location.

Methods: We retrospectively analyzed the CT data of the ASLAN001-003 study (NCT02338245). We analyzed TL size and number at each location focusing on therapeutic response from baseline to Week 12. We used TL diameter and volume to conduct an inter-arm comparison of the response according to: RECIST 1.1; stratified per TL location and; considering TLs independently. Multiple pairwise intra-arm comparisons of therapeutic responses were performed. Considering TL independently, weighted models were designed by adding weighted mean TL responses grouped by location. A sensitivity analysis tested the robustness of results.

Results: We evaluated 42 patients (88 TL) and 35 patients (74 TL) respectively at baseline and Week 12 .

We found reductions in breast TL burden in the VC arm compared to the LC arm ( $p=0.002$ (diameter), $\mathrm{p}<0.001$ (volume)). Responses and TL sizes at baseline were not correlated.

Explained variabilities of volume change per TL location, patient and patient:TL interaction were $36 \%$, $10 \%$ and $4 \%(\mathrm{VC})$, and $13 \%, 1 \%$ and $23 \%$, (LC).

A test of inter-arm difference of responses yielded $p=0.07$ (diameter), and $p<0.001$ (volume).

The sensitivity analysis confirmed our findings.

Conclusions: The therapeutic responses differed across locations; differential responses were drug-dependent. Stratified analysis provides better drug comparisons and is a powerful tool to understand TL heterogeneity.

\section{Background}

The Response Evaluation Criteria in Solid Tumors (RECIST) remain the most widely used criteria for assessing drug efficacy using imaging (1), primarily due to its simplicity and the lack of better established criteria (2). The heterogenous treatment responses observed in radiology following cytotoxic chemotherapy has already been reported (3). Now, some groups (4) (5) have raised concerns about RECIST that may be suboptimal for assessing treatment response to new generations of therapeutics like tyrosine kinase inhibitors (TKIs), whose mechanisms of action (MoAs) differ from that of chemotherapy.

Since 2010, the Food and Drug Administration (FDA) started considering new types of anticancer therapies (6) (7) for which the pattern of response was neither observed nor considered when RECIST 
were developed. Since then, radiology has not evolved at the same pace as these new treatments have emerged.

Molecular intratumor heterogeneity is often encountered with the use of TKIs, which rely on a novel MoA (8) (9), and categorizing a disease as stable is often evidence of drug effectiveness (10) (11).

Consequently, with new generations of anticancer treatments, patterns of radiology response may vary with tumor locations (12) (13), suggesting that sometimes, use of a stratified analysis would be more appropriate than a global one. Continued use of chemotherapy-based response criteria for assessing clinical efficacy of new therapies is therefore suboptimal (14). Similar limitations apply when assessing clinical efficacy of cocktails of drugs or response in basket trials.

In recent years, there have been rapid developments in the field of quantitative imaging in radiology, with the release of guidelines for qualification of quantitative imaging biomarkers (QIBs) (15) and recommendations for their implementation (16). Tumor volume in Computed Tomography (CT) has recently been presented as a valuable QIB that maxed all qualification steps (17).

Coupling tumor volume as an advanced QIB with a stratified analysis of the therapeutic response per disease location may offer useful insight into drug efficacy. In our study, we compared therapeutic response of Varlitinib + Capecitabine (VC) versus Lapatinib + Capecitabine (LC) in patients with human epidermal growth factor receptor 2 (HER2)-positive metastatic breast cancer (MBC) after trastuzumab therapy, using changes in tumor diameter and volume per tumor location.

\section{Materials And Methods}

The review boards of all participating institutions approved the study protocol. Written informed consent was not required as patient management was not impacted.

\section{Data collection}

We retrospectively analyzed CT scans measurements and annotations from the multicenter ASLAN001003 clinical trial (NCT02338245), which compared the therapeutic response of VC versus LC in patients with HER2-positive MBC after trastuzumab therapy. In the ASLAN001-003 trial, RECIST 1.1 were applied and, additionally, changes in sum of target lesion (TL) volume were monitored. The ASLAN001-003 trial used the LMS platform (Median Technologies, France) that automatically recorded tumor type, location, longest axial diameter (LAD), short axial diameter (SAD) and manually delineated volume.

Demographics and disease characteristics of the ASLAN001-003 trial are summarized in Table 1. The key inclusion criteria were:

1. Documented histological confirmation of breast cancer with HER2 overexpression or gene amplification (immunohistochemistry $3+$ or $2+$ with fluorescent/chromogenic/silver in situ hybridization+) prior to study entry. 
2. HER2 positive MBC that had progressed on prior firstline treatment with trastuzumab in metastatic setting or relapsed within 1 year of treatment with trastuzumab in adjuvant setting.

Table 1: Demographic and disease characteristics of the ASLAN001-003 clinical trial.

\begin{tabular}{|c|c|c|c|c|}
\hline \multicolumn{2}{|l|}{ Characteristic } & $\begin{array}{c}\text { Varlitinib + } \\
\text { Capecitabine } \\
(n=24)\end{array}$ & $\begin{array}{l}\text { Lapatinib + } \\
\text { Capecitabine } \\
(\mathbf{n}=26)\end{array}$ & $\begin{array}{l}\text { All Patients } \\
\qquad(\mathrm{N}=50)\end{array}$ \\
\hline \multicolumn{2}{|c|}{ Age, median (range) y } & $53.5(29-83)$ & $56.5(33-79)$ & $55.0(29-83)$ \\
\hline \multicolumn{2}{|c|}{ Female sex, No. (\%) } & $24(100)$ & $26(100)$ & $50(100)$ \\
\hline \multirow{4}{*}{$\begin{array}{l}\text { Ethnic origin, No. } \\
\text { (\%) }\end{array}$} & Asian-Chinese & $18(75.0)$ & $18(69.2)$ & $36(72.0)$ \\
\hline & Asian-other & $3(12.5)$ & $4(15.4)$ & $7(14.0)$ \\
\hline & White & $2(8.3)$ & $2(7.7)$ & $4(8.0)$ \\
\hline & Other & $1(4.2)$ & $2(7.7)$ & $3(6.0)$ \\
\hline \multirow{3}{*}{$\begin{array}{l}\text { ECOG } \\
\text { performance } \\
\text { status, No. (\%) }\end{array}$} & 0 & $19(79.2)$ & $20(76.9)$ & $39(78.0)$ \\
\hline & 1 & $3(12.5)$ & $6(23.1)$ & $9(18.0)$ \\
\hline & 2 & $2(8.3)$ & 0 & $2(4.0)$ \\
\hline \multirow{2}{*}{$\begin{array}{l}\text { Breast cancer } \\
\text { status, No. (\%) }\end{array}$} & Recurrence & $3(12.5)$ & $1(4.8)$ & $4(8.0)$ \\
\hline & Metastasis & $21(87.5)$ & $25(96.2)$ & $46(92.0)$ \\
\hline \multirow{4}{*}{$\begin{array}{l}\text { HER2 IHC, No. } \\
(\%)\end{array}$} & $1+$ & $1(4.2)$ & 0 & $1(2.0)$ \\
\hline & $2+$ & $6(25.0)$ & $9(34.6)$ & $15(30.0)$ \\
\hline & $3+$ & $14(58.3)$ & $17(65.4)$ & $31(62.0)$ \\
\hline & Missing & $3(12.5)$ & 0 & $3(6.0)$ \\
\hline \multirow{2}{*}{$\begin{array}{l}\text { HER2 FISH, No. } \\
\text { (\%) }\end{array}$} & Positive & $10(41.7)$ & $12(46.2)$ & $22(44.0)$ \\
\hline & $\begin{array}{l}\text { Not } \\
\text { performed }\end{array}$ & $14(58.3)$ & $14(53.8)$ & $28(56.0)$ \\
\hline
\end{tabular}

Abbreviations: $\mathrm{ECOG}=$ Eastern Cooperative Oncology Group; FISH = Fluorescence in situ hybridization; HER2 = Human epidermal growth factor receptor 2; IHC = Immunohistochemistry; $\mathrm{N}=$ Number of patients in the trial; $\mathrm{n}=$ Number of patients in the treatment arms; No. $=$ Number; $y=$ Years.

\section{Study workflow and analysis}

For our study, measurements and annotations were automatically retrieved from the original trial database. Measurements (LAD, SAD and volume) and annotations recorded at baseline and Week 12 were quality controlled and analyzed by a $15 \mathrm{Y}+$ medical imaging expert. Lymph nodes (LN) measurements (SAD) were specifically controlled to comply RECIST recommendations.

Our study plan was as follows: 


\section{Population statistics:}

We compared the tumor size and the number of tumors at each disease location, and for each treatment arm.

\section{Inter-arm comparison of the responses (Fig. 1):}

We analyzed the mean changes of the tumor burden (as \%) in considering:

1. The definition of tumor burden given by RECIST 1.1, where, for each patient, at each time point, the size (LAD/volume) of all TLs (up to 5 in number, independent of location, but no more than 2 per location) was summed, and these sums were monitored from baseline to Week 12;

2. Stratified tumor burden, where, for each patient, at each time point, the size (LAD/volume) of TLs from the same location were summed, and these sums were monitored from baseline to Week 12.

3. All tumors considered independent from patients and monitored from baseline to Week 12, with the average of tumor change in size (LAD/volume) computed per tumor location.

We tested if a significant relationship existed between tumor size at baseline and change from baseline at Week 12.

\section{Intra-arm comparison of the responses:}

In each arm, (independently to patients) we grouped tumors by location then we compared the average response (as \%) between these groups. We performed multiple pairwise comparisons of the responses between the various tumor locations (liver-breast, lung-breast, lymph node-breast, lung-liver, lymph nodeliver and lymph node-lung). In using either LAD (SAD in the case of $L N$ ) or volume, we tested for significant differences at Week 12 in each treatment arm. Finally, we computed the mean tumor changes by stratifying patients' responses and did an analysis of variance (ANOVA).

\section{Modeling of the stratified response:}

Considering tumors independently from patients, we designed a model by adding mean tumor responses, grouped by locations and weighted by the proportion of tumors at these locations. The weighted model summarized the response to treatment. The model will be computed for each arm to allows for a more accurate comparisons of inter-arm responses. Treatment responses were summarized as follows:

$$
\frac{1}{\sum\left(N b_{i}\right)} * \sum\left(N b_{i} * \overline{\Delta\left(T L_{l}\right)}\right)
$$

With:

$\mathrm{Nb}_{i}$ : Number of TLs at disease location ${ }_{i}(\mathrm{i}=$ breast, lung, liver or nodal tumors)

$\overline{\Delta\left(T L_{l}\right)}$ :

Mean change of TLs size (LAD or volume) at disease location ${ }_{i}$ 
Sensitivity analysis. We tested the robustness of our results by slightly changing the study input as follows (18):

1. Excluding patients exhibiting extreme treatment response at Week 12, then re-testing our conclusions with/without outliers;

2. Adjusting for the imbalance in number of independent tumors and number of patients after stratifying per tumor location at Week 12;

\section{Statistics}

The multiple comparisons of tumor sizes per tumor location were tested using Tukey Honest Significant Differences. Comparisons of tumor proportions at each location relied on a twosided Chi-square test. We computed waterfall plots of patients' response (summing all tumors for each patient), and in stratifying patients' response per tumor location, and Wilcoxon-rank tested the equivalence of interarm and stratified intra-arm responses.

Tests of multiple comparisons of tumor response per tumor location were performed applying Tukey Honest Significant Differences with adjustment of $p$-value.

We used a two-sided Chi-square test for evaluating inter-arm difference of response derived from the weighted models.

Eta-squared derived from the ANOVA reported the proportion of explained variabilities.

Data were considered as outliers when outside the 1.5 Inter Quartile Range (19).

The R 3.5.1 Cran software was used for statistics, $p<0.05$ was considered a significant difference.

\section{Results}

\section{Population statistics}

At baseline, 42 patients displayed at least $1 \mathrm{TL}$. A set of $88 \mathrm{TLs}$ was distributed per disease location as follows: lung $(31 \% n=27)$, breast $(26 \% n=23)$, liver $(23 \%, n=20)$, lymph nodes $(17 \% n=15)$ and miscellaneous $(3 \% ; n=3)$. Miscellaneous locations (skin and mediastinal lesions) were excluded as they were underrepresented. Therefore, 85 TLs were classified into 4 major groups by location (Table 2). To be noted that 22 patients had no visible primary breast tumors on CT due to previous trastuzumab treatment or because their tumors were visible only on mammography. 
Table 2

Proportion of tumors at each disease location at baseline

\begin{tabular}{|llllll|}
\hline & \multicolumn{2}{l}{ Varlitinib + Capecitabine } & \multicolumn{2}{l}{ Lapatinib + Capecitabine } & \multirow{2}{*}{ p value ${ }^{\mathbf{a}}$} \\
\cline { 1 - 5 } & Number of Tumors & $\%$ & Number of Tumors & $\%$ & \\
\hline Breast & 12 & 35.3 & 11 & 21.6 & 0.16 \\
\hline Lung & 7 & 20.6 & 20 & 39.2 & 0.07 \\
\hline Liver & 9 & 26.5 & 11 & 21.6 & 0.6 \\
\hline Lymph node & 6 & 16.6 & 9 & 17.6 & 1.0 \\
\hline Total & 34 & $\mathbf{1 0 0}$ & $\mathbf{5 1}$ & $\mathbf{1 0 0}$ & \\
\hline
\end{tabular}

a $p$ value corresponding to statistical significance of the inter-arm difference of proportion of tumor numbers, calculated using a two-sided Chi-square test.

At Week 12, 35 patients remained in the study (14 and 21 patients in the VC and LC arms, respectively) (Table 3 ) and 74 tumors were measured.

Table 3

Tumor burden changes from baseline to Week 12

\begin{tabular}{|lllll|}
\hline & $\begin{array}{l}\text { VC } \\
\text { (tumor } \\
\text { diameter) }\end{array}$ & $\begin{array}{l}\text { LC } \\
\text { (tumor } \\
\text { diameter) }\end{array}$ & $\begin{array}{l}\text { VC } \\
\text { (tumor } \\
\text { volume) }\end{array}$ & $\begin{array}{l}\text { LC } \\
\text { (tumor } \\
\text { volume) }\end{array}$ \\
\hline $\begin{array}{l}\text { Number of evaluable } \\
\text { patients }\end{array}$ & 14 & 21 & 14 & 21 \\
\hline $\begin{array}{l}\text { Mean change in tumor } \\
\text { burden }\end{array}$ & $-40.03 \%$ & $-21.19 \%$ & $-64.15 \%$ & $-25.59 \%$ \\
\hline p value $^{\text {b }}$ & 0.086 & & 0.13 & \\
\hline
\end{tabular}

LC = Lapatinib + Capecitabine; VC = Varlitinib + Capecitabine.

a Tumor burden computed as per Response Evaluation Criteria in Solid Tumor, by summing the size of up to 5 target tumors independent of tumor location, considering not more than 2 tumors per location.

${ }^{\mathrm{b}} \mathrm{p}$ value corresponding to statistical significance of inter-arm difference in mean changes in tumor burden (Wilcoxon rank test). 
Distributions of tumor size at baseline per tumor location in both treatment arms are displayed in Fig. 2 for both QIBs.

At baseline, there was no significant difference between the treatment arms in the proportion of tumors $(p=0.27)$, though there was a greater proportion of lung tumors in the LC arm versus the VC arm ( $p=$ 0.07 ) (Table 2). When considering either QIB, the mean size of breast tumors was significantly larger than that of tumors at the other locations $(p<0.002)$.

\section{Inter-arm comparison of the responses}

Tumor burden changes, in both treatment arms, are presented in Table 3. Waterfall plots of patient responses (LAD and volume) are displayed in Figs. 3; changes of tumor burden stratified per tumor location are in Figs. 4.

There were significant reductions in breast tumor burden in the VC arm compared to the LC arm $(p=0.002$ for $L A D, p<0.001$ for volume in favor of $V C$ arm). No significant interarm differences were noted for other TLs. Table 4 summarizes the mean tumor response with tumors considered independently from patients and grouped by location. Putting all tumors together without distinction from disease location and patient, a test of interarm difference of the response yielded $p=0.02$ for tumor LAD and $p=0.015$ for tumor volume. There was no significant relationship between the response and baseline tumor size by LAD or volume.

Table 4

Mean proportional change (\%) in diameter and volume of tumors considered independent of patients and grouped by disease locations

\begin{tabular}{|lllllll|}
\hline & \multicolumn{2}{l}{ Varlitinib + Capecitabine } & \multicolumn{2}{l|}{ Lapatinib + Capecitabine } & \multicolumn{2}{l|}{ p value $^{\text {a }}$} \\
\cline { 2 - 7 } & Diameter (\%) & Volume (\%) & Diameter (\%) & Volume (\%) & Diameter & Volume \\
\hline Breast & -50.19 & -85.06 & -16.15 & -30.90 & 0.001 & $<0.001$ \\
\hline Lung & -29.96 & -56.31 & -12.17 & 16.68 & 0.11 & 0.14 \\
\hline Liver & -8.64 & 7.24 & -21.55 & -44.71 & 0.80 & 0.40 \\
\hline Lymph node & -46.15 & -71.7 & -31.86 & -46.48 & 0.82 & 0.59 \\
\hline
\end{tabular}

a $p$ value corresponding to significance of the inter-arm comparison of the proportional change according to diameter and volume of tumors (Wilcoxon rank test).

\section{Intra-arm comparison of the responses}

Tables 5 and 6 summarizes, for tumor diameter and volume respectively, the difference of responses between the different pairs of tumor locations. 
Table 5

Intra-arm comparison of change in tumor diameter change at different tumor locations from baseline to Week 12

\begin{tabular}{|c|c|c|c|c|}
\hline \multirow{2}{*}{$\begin{array}{l}\text { Mean proportional change in tumor } \\
\text { diameter }\end{array}$} & \multicolumn{2}{|c|}{ Varlitinib + Capecitabine } & \multicolumn{2}{|c|}{ Lapatinib + Capecitabine } \\
\hline & $\begin{array}{l}\text { Difference }[95 \% \\
\text { Cls] }(\%)\end{array}$ & $\begin{array}{l}\mathrm{p} \\
\text { value }^{\mathrm{a}}\end{array}$ & $\begin{array}{l}\text { Difference [95\% } \\
\text { Cls] }(\%)\end{array}$ & $\begin{array}{l}\text { p } \\
\text { value }^{\mathrm{a}}\end{array}$ \\
\hline Liver-breast & $\begin{array}{l}49.96[-8.31 ; \\
108.29]\end{array}$ & 0.11 & $\begin{array}{l}-10.08[-70.42 ; \\
50.25]\end{array}$ & 0.97 \\
\hline Lung-breast & $\begin{array}{l}\text { 19.89 [-38.41; } \\
78.19]\end{array}$ & 0.77 & $\begin{array}{l}-1.76[-52.75 \\
49.23]\end{array}$ & 0.99 \\
\hline Lymph Node-breast & $6.33[-51.96 ; 64.63]$ & 0.99 & $\begin{array}{l}-16.01[-79.70 ; \\
47.67]\end{array}$ & 0.90 \\
\hline Lung-liver & $\begin{array}{l}-30.09[-98.69 \\
38.51]\end{array}$ & 0.61 & $8.32[-47.53 ; 64.18]$ & 0.98 \\
\hline Lymph node-liver & $\begin{array}{l}-43.65 \text { [-112.26; } \\
24.95]\end{array}$ & 0.30 & $\begin{array}{l}-5.93[-73.58 \\
61.71]\end{array}$ & 0.99 \\
\hline Lymph node-lung & $\begin{array}{l}-13.56 \text { [-82.16; } \\
55.03]\end{array}$ & 0.94 & $\begin{array}{l}-14.25[-73.72 ; \\
45.21]\end{array}$ & 0.91 \\
\hline
\end{tabular}

$\mathrm{Cl}=$ Confidence interval.

${ }^{a} p$ value corresponding to the test of a significant difference in the responses between the tumor locations (Test of Tukey Honest Significant Differences). 
Table 6

Intra-arm comparison of change in tumor volume at different tumor locations from baseline to Week 12

\begin{tabular}{|c|c|c|c|c|}
\hline \multirow{2}{*}{$\begin{array}{l}\text { Mean proportional change in } \\
\text { volume }\end{array}$} & \multicolumn{2}{|c|}{ Varlitinib + Capecitabine } & \multicolumn{2}{|c|}{ Lapatinib + Capecitabine } \\
\hline & $\begin{array}{l}\text { Difference }[95 \% \mathrm{Cls}] \\
(\%)\end{array}$ & $\begin{array}{l}\mathrm{p} \\
\text { value }^{\mathrm{a}}\end{array}$ & $\begin{array}{l}\text { Difference [95\% Cls] } \\
(\%)\end{array}$ & $\begin{array}{l}\mathrm{p} \\
\text { value }\end{array}$ \\
\hline Liver-breast & $\begin{array}{l}\text { 107.49 [26.34; } \\
\text { 188.64] }\end{array}$ & 0.007 & $\begin{array}{l}-21.09[-126.18 \\
83.98]\end{array}$ & 0.94 \\
\hline Lung-breast & $\begin{array}{l}30.49[-50.67 \\
111.64]\end{array}$ & 0.71 & $\begin{array}{l}42.11[-48.31 ; \\
132.52]\end{array}$ & 0.58 \\
\hline Lymph Node-breast & $14.47[-66.68 ; 95.62]$ & 0.96 & $\begin{array}{l}-17.16[-128.08 \\
93.77]\end{array}$ & 0.97 \\
\hline Lung-liver & $\begin{array}{l}-77.00[-172.49 \\
18.49]\end{array}$ & 0.14 & $\begin{array}{l}63.20[-35.55 ; \\
161.96]\end{array}$ & 0.32 \\
\hline Lymph node-liver & $-93.02[-188.51 ; 2.47]$ & 0.057 & $\begin{array}{l}3.94[-113.88 \\
121.77]\end{array}$ & 0.99 \\
\hline Lymph Node-lung & $\begin{array}{l}-16.01[-111.51 ; \\
79.48]\end{array}$ & 0.96 & $\begin{array}{l}-59.26[-164.21 \\
45.69]\end{array}$ & 0.42 \\
\hline
\end{tabular}

$\mathrm{Cl}=$ Confidence interval.

a $p$ value of the test of a significant difference in the responses between the tumor locations (Test of Tukey Honest Significant Differences).

For changes in tumor diameter (Table 5), explained variabilities per tumor location, patient and patient:tumor interaction were $22 \%, 5 \%$ and $16 \%$, respectively, in the VC arm, and $2 \%, 0.5 \%$ and $30 \%$, respectively, in the LC arm.

For changes in tumor volume (Table 6), explained variabilities per tumor location, patient and patient:tumor interaction were $36 \%, 10 \%$ and $4 \%$, respectively, in the VC arm, and $13 \%, 1 \%$ and $23 \%$, respectively, in the LC arm.

\section{Model design}

We applied our model using the distribution of tumor location (Table 2) and the average response by tumor location (independent tumors) in Table 5 and Table 6. Thus, we modeled the response to treatment for the VC and LC arms respectively in Eqs. 1 and 2 (using LAD) and respectively in Eqs. 3 and 4 (using volume).

$1 / 28 *(11 *-50.19 \%+5 *-29.96 \%+6 *-8.64 \%+6 *-46.15 \%)=-36.8 \% \quad$ Eq. 1 
Volume

$1 / 28 *(11 *-85.06 \%+5 *-56.31 \%+6 * 7.23 \%+6 *-71.7 \%)=-57.3 \% \quad$ Eq. 3

$1 / 46 *(11 *-30.90 \%+18 * 16.68 \%+11 *-44.71 \%+6 *-46.48 \%)=-17.6 \% \quad$ Eq. 4

a test for a significant difference in inter-arm responses yielded $p=0.07$ (using LAD), and $p<0.001$ (using volume) both in favor of VC arm.

\section{Sensitivity analysis}

The inter-arm comparison of the stratified responses yielded $p=0.015$ (using LAD) and $p=0.03$ (using volume) after removing outliers at Week 12 ( $n=6$ for tumor diameter, $n=3$ for tumor volume) (supplementary Table I.1). When considering each tumor independently from patients, interarm comparison yielded $p<0.007$ (for tumor diameter) and $p=0.016$ (for tumor volume) after removing outliers ( $n=7$ for tumor diameter, $n=4$ for tumor volume). Intra-arm comparisons of the stratified responses by disease location are summarized in supplementary Tables I.2 and I.3.

Equations 1.1 to 1.4 (electronic supplementary material [ESM]) obtained following data adjustment at Week 12 with balancing of tumors at each disease location were comparable to equations 1 to 4 . A test for a significant difference in inter-arm responses yielded $p=0.17$ (tumor diameter) and $p=0.003$ (tumor volume).

Equations 1.5 to 1.8 (electronic supplementary material [ESM]) obtained in computing stratified change of tumor burden, balancing numbers of patients having tumor at the same location, were comparable to equations 1 to 4 . A test for a significant difference in inter-arm responses yielded $p=0.26$ for tumor diameter and $p=0.11$ for tumor volume in favor of $\mathrm{VC}$ arm.

\section{Discussion}

Our study showed that breast tumors were, on average, significantly larger other tumors $(p<0.001)$. There was no significant interarm difference in the proportion of tumors at different disease locations, though there was a greater proportion of lung tumors in the LC arm $(p=0.07)$. Interarm tests showed a trend towards superiority of the $\mathrm{VC}$ arm per patient, and confirmed superiority of the $\mathrm{VC}$ arm when tumors were considered independently. Multiple intra-arm comparisons showed that tumor volume is more sensitive than LAD for detection of differential responses at different disease locations. In the VC arm, we found a significant differential response between breast and liver tumors using volume $(p=0.007)$ and a trend towards superiority using volume in differential response for lymph node vs. liver tumors $(p=0.057)$. No significant differences were measured in the LC arm using LAD or volume.

Results of the intra-arm multiple comparisons confirmed the stratified inter-arm results, showing a more favorable response in the VC arm compared to the LC arm, for both QIBs ( $p=0.07$ for LAD, $p<0.001$ for 
volume). These results were also confirmed by the inter-arm comparisons of the weighted models and the ANOVA, indicating a greater variability per tumor locations in the VC arm. The results of our study are strengthened by a sensitivity analysis that reported no significant impact of outliers upon our conclusions, and no change in the stratified responses of VC over LC, after adjusting the proportion of TLs at each disease location. Our stratified analysis showed the effectiveness of the drug at specific disease location. This insight would help to improve drug indications and to design more effective drug combinations.

Researchers have reported differential responses according to disease location. Menzies et al (20) found significantly different Time To Best Response for subcutaneous soft tissue and lung metastases compared to lymph node and liver metastases, and Crusz et al (21) found that $55.6 \%$ of patients showed a heterogeneous response. These studies drew contradictory conclusions regarding a relationship between tumor size at baseline and response. Our study did not show a relationship between tumor size at baseline and response. Usually, tumors have complex shapes and are heterogeneous; volumetric measurements have long showed better precision and accuracy than linear measurement, notably in advanced lung cancer patients (17) (21). However, very few studies have proved that changes in tumor volume better correlate to the disease or can be an alternative for clinical trial. In our study, we found that when tumor volumes were used, $\mathrm{p}$ values were lower when testing inter-arm response according to a weighted model of stratified response $p=0.07$ (for tumor diameter), and $p<0.001$ (for tumor volume) or when tumors were all considered as independent $p=0.02$ (for tumor diameter) and $p=0.015$ (for tumor volume). We also found that tumor volume was more discriminant than diameter when testing differential response (e.g. $p=0.007$ for liver-breast in VC arm). Similar discrimination was not observed with RECIST ( $p=0.13$ for volume), $(p=0.086$ for diameter). This can be explained by the design of RECIST that recommend adding tumors from different location, therefore losing the benefit of the volume.

We can also consider that the stratification of imaging therapeutic response represents a mean of investigation per say (22). It is known that spatial and temporal tumor heterogeneity can be due to the mutational status of tissues, their cellular morphology, metabolism, and proliferative and metastatic potential (23). Therapeutic response stratification can therefore be seen as an indirect noninvasive feedback on tumor heterogeneity. More specifically, the temporal monitoring of clinical data coupled with stratified responses could inform about different resistance mechanisms and their outbreak (24). Enriching biological data with stratified imaging responses would help to understand the MoA, identify drug sensitive or resistant cells and investigate new targeted therapy approaches.

Our study had some limitations, the first being that we analyzed tumor response over a short period of time. To match the ASLAN003-001 trial setting, we restricted our analysis to Week 12. We may hypothesize that the stratified response at each tumor location can vary over time. For instance, at treatment onset, a drug could exhibit a superior efficacy upon primary breast tumors compared to metastases, which could fade, disappear, or even reverse over time. Because of the limited dataset we could not extend our study over multiple time points. A second limitation of our study was that we did not 
consider the aspect of measurement reliability. In our dataset, tumors had different size distributions according to locations, and the proportions of tumors at various locations differed slightly between the arms. Several groups have investigated the measurement reliability according to tumor size and location (25) (26). A more sophisticated model of stratified response would include the reliability of measurements as a parameter. A third limitation of our study was that it was not possible to consider all RECIST aspects as the unequivocal appearance of new lesions and progression of nontarget lesions (nTLs). In our study, at Week 12, a single new unequivocal new lesion was detected, 2 nTLs progressed while 9 decreased. The small data sizing precluded any significant conclusions.

A fourth limitation is inherent to the ASLAN003-001 trial that mainly included Asian patients while Wagner et al. (27) reminded that different response may exists between Asian and Caucasian ethnicities. Therefore, the generalizability of our observations needs to be confirmed with non-Asian cohorts.

\section{Conclusion}

We found that drugs have different efficacy across tumor locations. In the era of new therapies, stratified analysis of response will provide better assessments and drug comparisons, and be a powerful tool contributing to improved understanding of the MoA behind tumor heterogeneity.

\section{Abbreviations}




\begin{tabular}{|ll|}
\hline ANOVA & Analysis of variance \\
\hline CAP & Chest/abdomen pelvis \\
\hline CT & Computed tomography \\
\hline ESM & Electronic supplementary material \\
\hline FDA & Food and Drug Administration \\
\hline HER2 & Human epidermal growth factor receptor 2 \\
\hline LAD & Longest Axial Diameter \\
\hline LC & Lapatinib plus Capecitabine \\
\hline LN & Lymph Node \\
\hline MBC & Metastatic breast cancer \\
\hline MoA & Mechanism of action \\
\hline nTL & Non-target lesion \\
\hline QIB & Qualitative imaging biomarker \\
\hline RECIST & Response Evaluation Criteria in Solid Tumor \\
\hline SAD & Shortest Axial Diameter \\
\hline TKI & Tyrosine kinase inhibitor \\
\hline TL & Target lesion \\
\hline VC & Varlitinib plus Capecitabine \\
\hline
\end{tabular}

\section{Declarations}

\section{Ethics approval and consent to participate}

Our retrospective study did not include interaction or intervention with human subjects or include any access to identifiable private information, no IRB approval was required.

Written informed consent was not required for this study because not impacting patient management.

\section{Consent for publication}

We certify that this manuscript is not under consideration for publication elsewhere. All co-authors take public responsibility for the content of the present manuscript. The final version of the manuscript has been reviewed and approved by all co-authors.

\section{Availability of data and materials}


Study subjects or cohorts have been previously reported in ASLAN001-003 clinical trial (NCT02338245).

\section{Competing interest}

The authors of this manuscript, Hubert Beaumont, Catherine Klifa, Nathalie Faye declare relationships with the following companies: Median Technologies.

Chih-Yi Hsieh declare relationships with ASLAN Pharmaceuticals

Other authors of this manuscript declare no relationships with any companies, whose products or services may be related to the subject matter of the article.

\section{Funding}

This study did not received funding.

\section{Authors' contributions}

We certify that all co-authors contributed equally and significantly to the study and to the design of the manuscript

\section{Acknowledgements}

We acknowledge Nicolas Dano for his constant support to the study and Souhil Zaim for his valuable advises.

\section{References}

1. Eisenhauer E a., Therasse P, Bogaerts J, Schwartz LH, Sargent D, Ford R, et al. New response evaluation criteria in solid tumours: Revised RECIST guideline (version 1.1). Eur J Cancer [Internet]. 2009;45(2):228-47. Available from: http://dx.doi.org/10.1016/j.ejca.2008.10.026

2. Fojo AT, Noonan A, Sharma MR, Maitland ML, Ratain MJ. Why RECIST works and why it should stay Counterpoint. Cancer Res. 2012;72(20):5151-8.

3. van Kessel CS, Samim M, Koopman M, van den Bosch MAAJ, Borel Rinkes IHM, Punt CJA, et al. Radiological heterogeneity in response to chemotherapy is associated with poor survival in patients with colorectal liver metastases. Eur J Cancer [Internet]. 2013 Jul;49(11):2486-93. Available from: https://linkinghub.elsevier.com/retrieve/pii/S095980491300261X

4. Nishino M. Tumor Response Assessment for Precision Cancer Therapy: Response Evaluation Criteria in Solid Tumors and Beyond. Am Soc Clin Oncol Educ B. 2018;(38):1019-29.

5. Nishino M, Dahlberg SE, Cardarella S, Jackman DM, Rabin MS, Hatabu H, et al. Tumor volume decrease at 8 weeks is associated with longer survival in EGFR-mutant advanced non-small-cell lung cancer patients treated with EGFR TKI. J Thorac Oncol [Internet]. 2013;8(8):1059-68. Available from: http://dx.doi.org/10.1097/JT0.0b013e318294c909 
6. Woodcock J, US Food and Drug Administration. AstraZeneca Pharmaceuticals LP: Withdrawal of Approval of a New Drug Application for IRESSA [Internet]. 2012. Available from: https://www.federalregister.gov/documents/2012/04/25/2012-9944/astrazeneca-pharmaceuticalsIp-withdrawal-of-approval-of-a-new-drug-application-for-iressa

7. Wei SC, Duffy CR, Allison JP. Fundamental mechanisms of immune checkpoint blockade therapy. Cancer Discov. 2018;8(9):1069-86.

8. Segovia-Mendoza M, González-González ME, Barrera D, Díaz L, García-Becerra R. Efficacy and mechanism of action of the tyrosine kinase inhibitors gefitinib, lapatinib and neratinib in the treatment of her2-positive breast cancer: Preclinical and clinical evidence. Am J Cancer Res. 2015;5(9):2531-61.

9. Krajewski KM, Braschi-Amirfarzan M, DiPiro PJ, Jagannathan JP, Shinagare AB. Molecular targeted therapy in modern oncology: Imaging assessment of treatment response and toxicities. Korean $\mathrm{J}$ Radiol. 2017;18(1):28-41.

10. Crusz SM, Tang YZ, Sarker SJ, Prevoo W, Kiyani I, Beltran L, et al. Heterogeneous response and progression patterns reveal phenotypic heterogeneity of tyrosine kinase inhibitor response in metastatic renal cell carcinoma. BMC Med [Internet]. 2016;14(1):1-9. Available from: http://dx.doi.org/10.1186/s12916-016-0729-9

11. Tolcher AW. Stable disease is a valid end point in clinical trials. Cancer J [Internet]. 2009 [cited 2014 Jan 15];15(5):374-8. Available from: http://www.ncbi.nlm.nih.gov/pubmed/19826356

12. Lee K-W, Bang S-M, Lee JS, Kim D-W, Kim J-S, Kim YJ, et al. Different metastatic pattern according to the KRAS mutational status and site-specific discordance of KRAS status in patients with colorectal cancer. BMC Cancer. 2012;12(1).

13. Callea M, Albiges L, Gupta M, Cheng S, Elizabeth M, Song J, et al. Differential Expression of PD-L1 between Primary and Metastatic Sites in Clear-Cell Renal Cell Carcinoma. 2015;3(10):1158-64.

14. Khoja L, Kibiro M, Metser U, Gedye C, Hogg D, Butler MO, et al. Patterns of response to anti-PD-1 treatment: An exploratory comparison of four radiological response criteria and associations with overall survival in metastatic melanoma patients. Br J Cancer [Internet]. 2016;115(10):1186-92. Available from: http://dx.doi.org/10.1038/bjc.2016.308

15. O'Connor JPB, Aboagye EO, Adams JE, Aerts HJWL, Barrington SF, Beer AJ, et al. Imaging biomarker roadmap for cancer studies. Nat Rev Clin Oncol [Internet]. 2017 Mar 11;14(3):169-86. Available from: http://www.nature.com/articles/nrclinonc.2016.162

16. Shukla-Dave A, Obuchowski NA, Chenevert TL, Jambawalikar S, Schwartz LH, Malyarenko D, et al. Quantitative imaging biomarkers alliance (QIBA) recommendations for improved precision of DWI and DCE-MRI derived biomarkers in multicenter oncology trials. J Magn Reson Imaging [Internet]. 2019 Jun 19;49(7):e101-21. Available from: https://onlinelibrary.wiley.com/doi/abs/10.1002/jmri.26518

17. Mozley PD, Bendtsen C, Zhao B, Schwartz LH, Thorn M, Rong Y, et al. Measurement of tumor volumes improves RECIST-based response assessments in advanced lung cancer. Transl Oncol 
[Internet]. 2012 Feb [cited 2014 Jan 15];5(1):19-25. Available from:

http://www.pubmedcentral.nih.gov/articlerender.fcgi?

artid $=3281412 \&$ tool $=$ pmcentrez\&rendertype=abstract

18. Thabane L, Mbuagbaw L, Zhang S, Samaan Z, Marcucci M, Ye C, et al. A tutorial on sensitivity analyses in clinical trials: The what, why, when and how. BMC Med Res Methodol. 2013;13(1).

19. Exploratory Data Analysis BT - The Concise Encyclopedia of Statistics. In New York, NY: Springer New York; 2008. p. 192-4. Available from: https://doi.org/10.1007/978-0-387-32833-1_136

20. Menzies AM, Haydu LE, Carlino MS, Azer MWF, Carr PJA, Kefford RF, et al. Inter- And intra-patient heterogeneity of response and progression to targeted therapy in metastatic melanoma. PLoS One. 2014;9(1):1-9.

21. Cai W-L, Hong G-B. Quantitative image analysis for evaluation of tumor response in clinical oncology. Chronic Dis Transl Med [Internet]. 2018;4(1):18-28. Available from: https://doi.org/10.1016/j.cdtm.2018.01.002

22. Diaz-Cano SJ. Tumor heterogeneity: Mechanisms and bases for a reliable application of molecular marker design. Int J Mol Sci. 2012;13(2):1951-2011.

23. Martelotto LG, Ng CKY, Piscuoglio S, Weigelt B, Reis-Filho JS. Breast cancer intra-tumor heterogeneity. Breast Cancer Res. 2014;16(3):1-11.

24. Norouzi S, Gorgi Valokala M, Mosaffa F, Zirak MR, Zamani P, Behravan J. Crosstalk in cancer resistance and metastasis. Crit Rev Oncol Hematol [Internet]. 2018 Dec;132:145-53. Available from: https://linkinghub.elsevier.com/retrieve/pii/S1040842818301999

25. McErlean A, Panicek DM, Zabor EC, Moskowitz CS, Bitar R, Motzer RJ, et al. Intra- and Interobserver Variability in CT Measurements in Oncology. Radiology [Internet]. 2013 Nov;269(2):451-9. Available from: http://pubs.rsna.org/doi/10.1148/radiol.13122665

26. Pupulim LF, Ronot M, Paradis V. Volumetric measurement of hepatic tumors: Accuracy of manual contouring using CT with volumetric pathology as the. Diagn Interv Imaging [Internet]. 2018;99(2):83-9. Available from: http://dx.doi.org/10.1016/j.diii.2017.11.002

27. Wagner AD, Grabsch HI, Mauer M, Marreaud S, Caballero C, Thuss-Patience P, et al. EORTC-1203GITCG - The "INNOVATION"-trial: Effect of chemotherapy alone versus chemotherapy plus trastuzumab, versus chemotherapy plus trastuzumab plus pertuzumab, in the perioperative treatment of HER2 positive, gastric and gastroesophageal junction aden. BMC Cancer. 2019;19(1):19.

\section{Figures}


1)
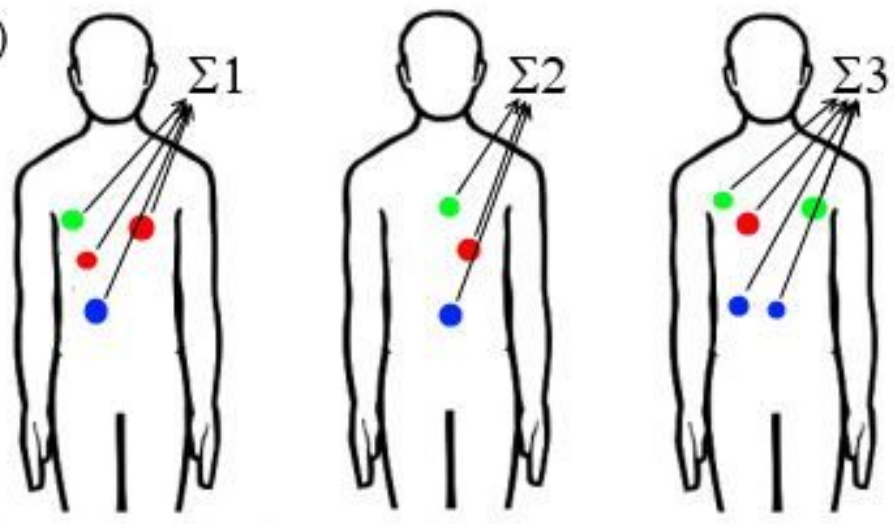

Patient 1

Patient 2

Patient 3 Patient N
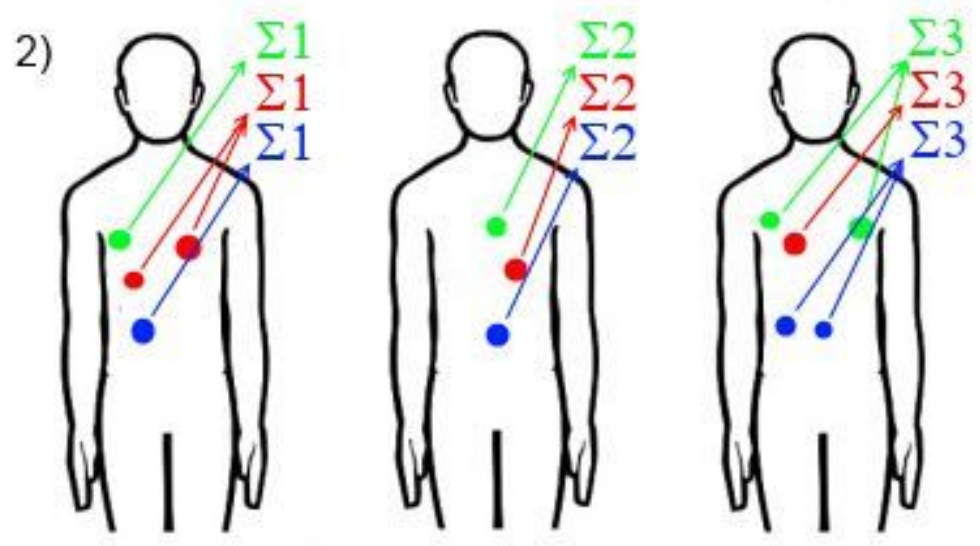

Patient 1

Patient 2

Patient 3

Patient N

3)

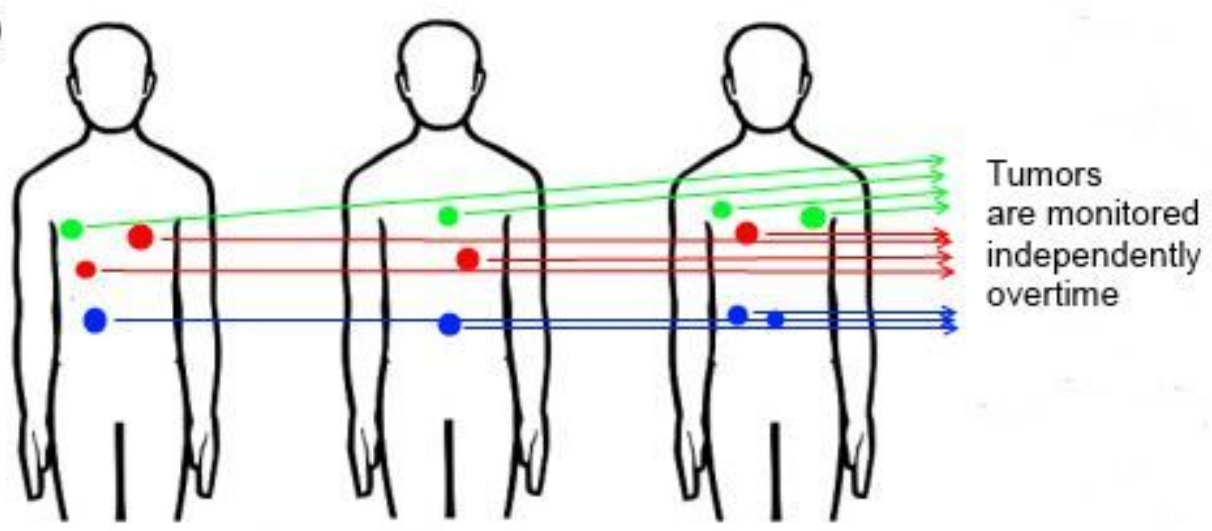

Patient 1

Patient 2

Patient 3

Patient $\mathrm{N}$

Figure 1

Inter-arm comparisons of tumor responses. Three different analyses were performed. 1) Patient tumor burden was monitored according to RECIST 1.1. At each time point, for a given patient, the size of all target tumors was summed, and these sums were monitored over time. 2) Patient tumor burden was stratified by tumor location. At each time point, for a given patient, the size of target tumors from the same location was summed, and these sums were monitored over time. 3) All tumors were considered as 
independent from patients and monitored independently over time; the mean tumor change was computed per location.
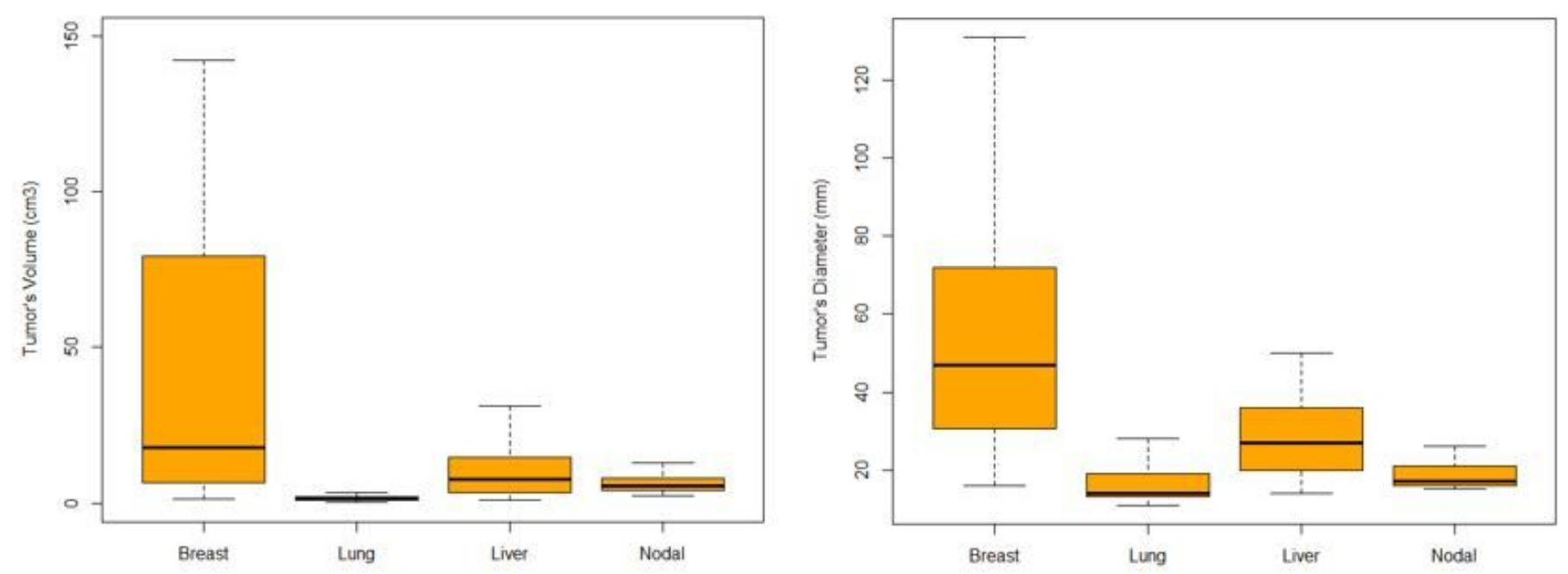

Figure 2

Boxplots showing distribution of tumor size at baseline per location of the disease (breast, lung, liver and nodal) with tumor volume $(\mathrm{cm} 3)$ on the left and tumor diameter $(\mathrm{mm})$ on the right. Beast tumors were larger, $\mathrm{P}<0.001$ for volume or diameter using Tukey Honest Significant Difference test
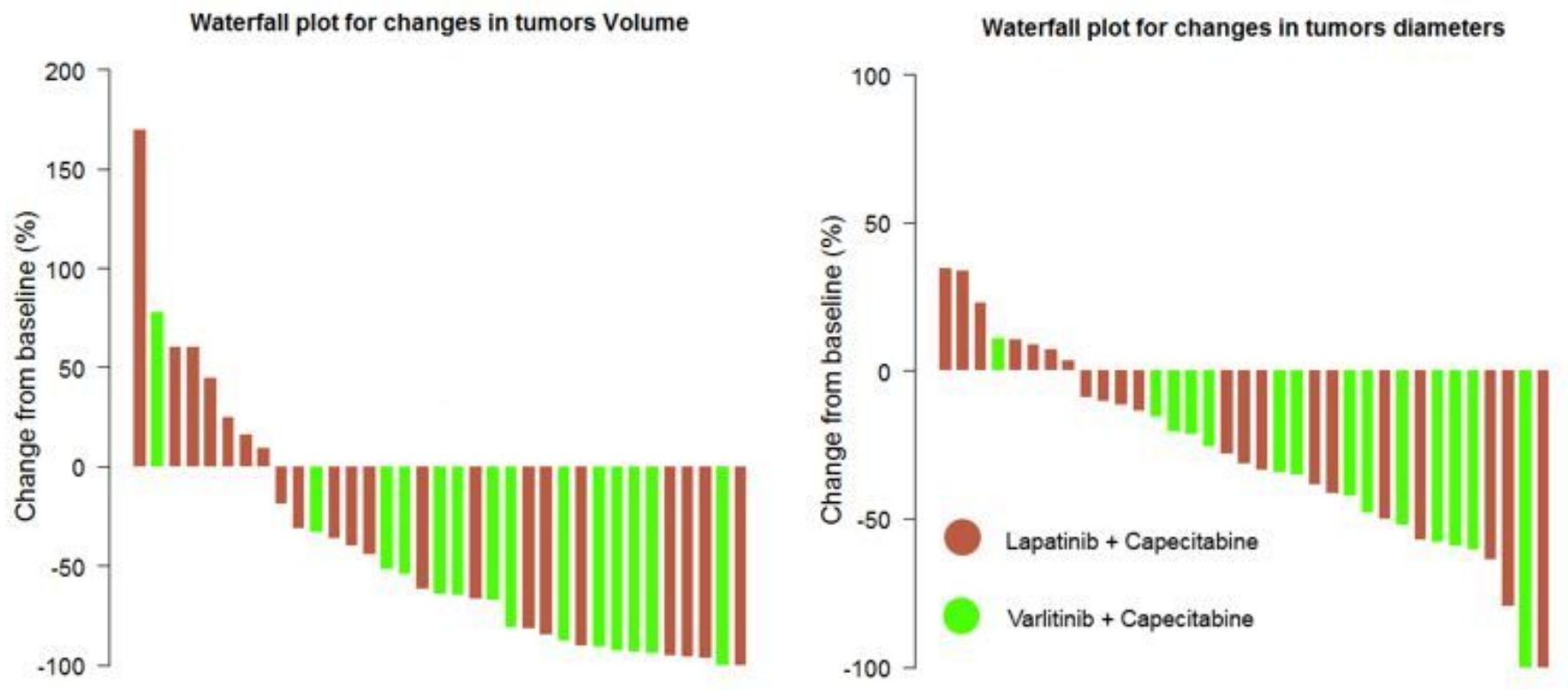

Figure 3 
Waterfall plot showing changes from baseline at Week 12 in tumor volume (on left) and tumor diameter (on right). Green bars represent responses in the Varlitinib + Capecitabine arm; red bars represent responses in the Lapatinib + Capecitabine arm.
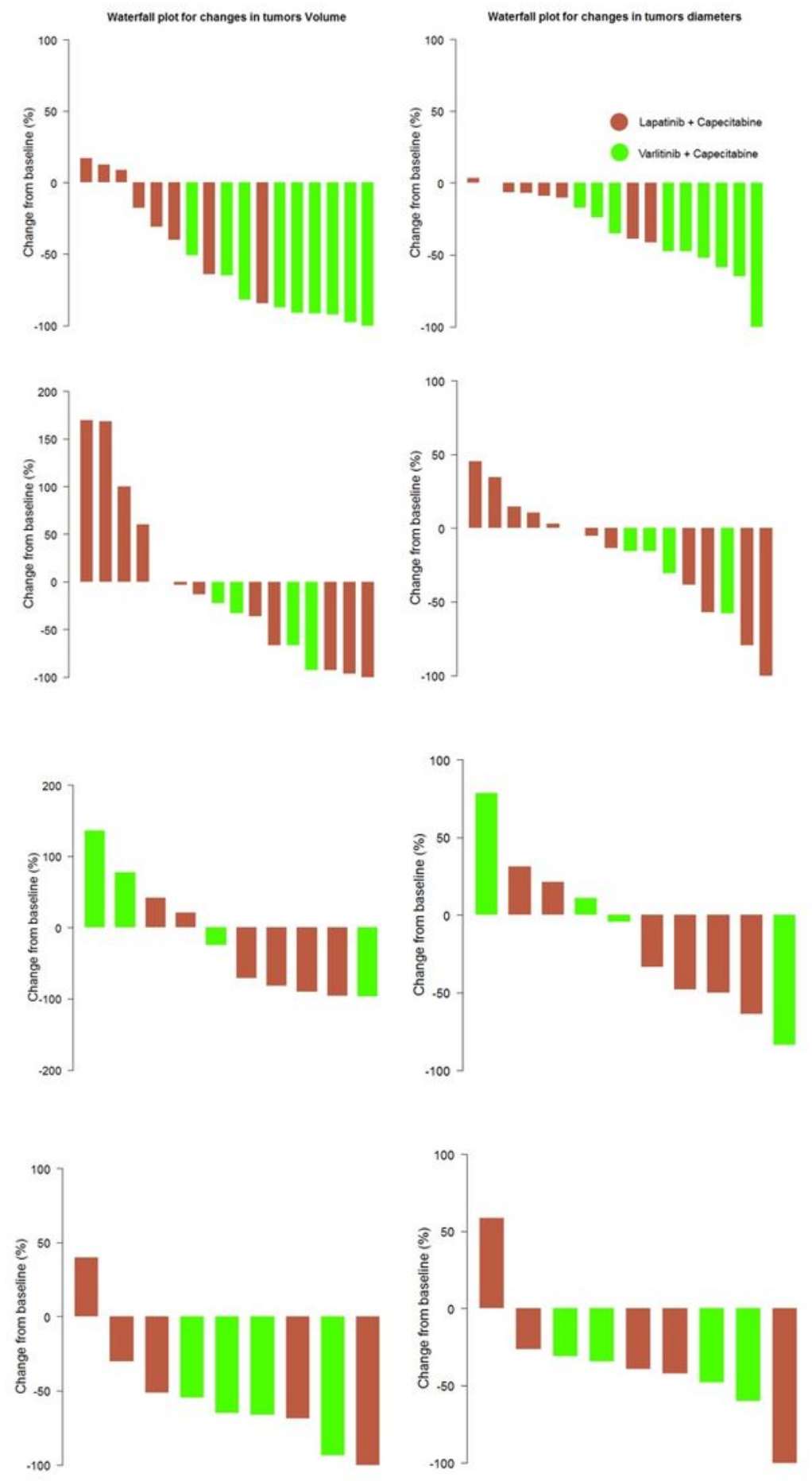

Figure 4

Waterfall plot showing stratified changes from baseline to Week 12 in tumor volume (on right) and diameter (on left) for breast, lung, liver and lymph node tumors (from top to bottom). Green bars represent 
responses in the Varlitinib + Capecitabine arm; red bars represent responses in the Lapatinib + Capecitabine arm. There was significant inter arm difference only for changes in breast tumor burden $(p=0.002$ and $p<0.001$, respectively, for tumor diameter and volume as qualitative imaging biomarkers).

\section{Supplementary Files}

This is a list of supplementary files associated with this preprint. Click to download.

- ELECTRONICSUPPLEMENTARYMATERIAL.docx

- AnnexesTargetedtherapies.docx 